\title{
Myelodysplastic syndrome (MDS) associated with EBV infection in a pediatric patient
}

\author{
Esther Manor $^{1 *}$, Yonat Shemer-Avni ${ }^{2}$, Sophia Boriakovski ${ }^{1}$, Michael Kafka ${ }^{3}$, Lipa Bodner ${ }^{4}$, \\ Joseph Kapelushnik ${ }^{5}$ \\ ${ }^{1}$ Soroka Medical Center, Faculty of Health Sciences, Institute of Human Genetics, Ben-Gurion University of the Negev, Beer-Sheva, \\ Israel \\ ${ }^{2}$ Soroka Medical Center, Laboratory for Viral Diagnosis, Faculty of Health Sciences, Ben-Gurion University of the Negev, Beer-Sheva, \\ Israel \\ ${ }^{3}$ Soroka Medical Center, Department of Hematology, Faculty of Health Sciences, Ben-Gurion University of the Negev, Beer-Sheva, \\ Israel \\ ${ }^{4}$ Soroka Medical Center, Department of Oral and Maxillofacial Surgery, Faculty of Health Sciences, Ben-Gurion University of the \\ Negev, Beer-Sheva, Israel \\ ${ }^{5}$ Soroka Medical Center, Department of Pediatric Hemato-Oncology, Faculty of Health Sciences, Ben-Gurion University of the Negev, \\ Beer-Sheva, Israel \\ Email: ${ }^{*}$ manore@bgu.ac.il
}

Received 30 December 2012; revised 31 January 2013; accepted 10 February 2013

\begin{abstract}
Background: Epstein Barr Virus (EBV) is associated with different kinds of tumors. In the present study we tried to understand its role in pediatric MDS of a 4-year-old girl with EBV infection and MDS with refractory anemia and monosomy 7 . Procedures: The work-up included: hematological tests; serology for IgM, IgG antibodies to EBV; PCR for EBV; cytogenetics of bone marrow (BM), and FISH analysis of BM and blood; immunohistochemistry-LMP1 expression on BM smears. Results: Hematological follow up showed constant mild dysplastic changes mostly in the erythroid lineage. PCR for EBV showed positive results in the nasopharygeal smears as well as in blood 15 weeks after disease onset. Cytogenetic analysis showed monosomy 7 in all the mitoses of BM sample. Fluorescence in situ hybridization (FISH) showed monosomy $\mathbf{7 \%}$ in $57 \%$ of the cells, followed by a decreased tendency in the percentage of monosomy 7 cells in both BM and blood. Immunohistochemistry for EBV-latent membrane protein 1 (LMP-1) on the patient's BM smears, 21 weeks post disease onset, showed $9 \%$ positive cells, $80 \%$ of them carried monosomy 7. Conclusion: The parallel occurrence of the EBV infection and MDS, as well as the continuous EBV PCR positive and monosomy 7, support the possibility that they are related.
\end{abstract}

Keywords: Pediatric; MDS; EBV Infection

\footnotetext{
*Corresponding author.
}

\section{INTRODUCTION}

Myelodysplastic syndrome (MDS) represents a heterogeneous clonal hematopoetic stem cell disorder characterized by ineffective hematopoiesis and increased risk of transformation to MDS-related leukemia. MDS is uncommon in children and accounts for less than $5 \%$ of all hematopoetic neoplasms [1].

Monosomy 7 is the most common chromosomal aberration in MDS [2-6]. It implies a rather poor prognosis that is associated with high risk of transformation to acute leukemia [7]. The age distribution is $2-5$ years with male predominance of $70 \%-80 \%$ [8]. There are also reports on familial MDS with monosomy 7. Familial monosomy 7 is defined when at least two siblings are found to carry monosomy 7 associated with pre-leukemic or leukemic manifestations [9].

Epstein Barr Virus (EBV) is one of the causative agents of infectious mononucleosis and the first described human tumor virus. EBV was discovered in Burkitt's lymphoma tumor cells, characterized by $\mathrm{t}(8,14)$, $\mathrm{t}(2,8)$, and $\mathrm{t}(8,22)[10]$. It has also been found to be associated with post-transplant lymphoproliferative disorders (PTLD) and with malignancies such as, Hodgkin's lymphoma (HD) and nasopharyngeal carcinoma (NPC) $[11,12]$.

EBV's latent membrane protein (LMP1) gene is considered the main viral oncogene driving cell growth, promoting metastases, apoptotic resistance, and immune modulation, including blockade of interferon alpha-induced antiviral signaling [13].

Infection with EBV can produce symptoms closely resembling those seen in patients with MDS. This raises 
a question regarding the diagnosis of malignant disease in cases of MDS in children with EBV infection [14-16] and the role of EBV in MDS pathogenesis: Does EBV infection mimic childhood MDS? Does it trigger or enhance childhood MDS onset? Is EBV the etiology of MDS or is MDS onset with EBV infection just coincidental?

Here we present results that might support the possibility that EBV plays a role in pediatric MDS of a 4year-old girl with monosomy 7 .

\section{CASE REPORT AND RESULTS}

A 4-year-old girl was admitted to the pediatric department with suspected anemia, after experiencing two episodes of fever about 3 weeks earlier. Upon admittance she was weak and pale with a diagnosis of infectious mononucleosis by the family doctor, although no tests were undertaken. According to her mother's anamnesis, she had been suffering on and off from viral and bacterial infections.

Hematology: Peripheral blood count showed a white blood count (WBC) of $4400 / \mu 1$ with no blasts, Hb of 4.9 $\mathrm{g} / \mathrm{dl}$, and platelet count of $175,000 / \mu \mathrm{l}$. The clinical impression was of erythroblastopenia of childhood, probably related to viral infection. Serial transfusions of packed cells elevated the $\mathrm{Hb}$ to $6.4 \mathrm{~g} / \mathrm{dl}$. Peripheral blood count was followed and transfusions of packed cells were given due to the anemia.

Serology: The work-up revealed positive antibodies to cytomegalovirus (CMV) IgM and IgG, with high avidity. Antibodies to EBV (viral capsid antigen) VCA IgG and IgM were positive; however, IgG antibodies to EBV nuclear antigen (EBNA) were negative. These results indicate that the patient was suffering from an acute primary EBV infection with possible CMV reactivation. EBV acute infection was further confirmed by EBV-positive PCR results of the pharyngeal smear.

Cytogenetics: Cytogenetic analysis of bone marrow (BM) was performed according to standard methods. BM cells were cultured for 24 hours using methotrexate for synchronization. About 15 metaphases were analyzed using G-band staining. Karyotypes were assigned according to the International System for Human Cytogenetic Nomenclature (ISCN 2009) guidelines [17].

FISH analysis was performed according to the manufacturer's instructions using an LSI D7S522 (7q31) spectrum orange/CEP spectrum green (VYSIS) probe. More than 500 cells were analyzed.

Virology tests: IgM and IgG antibodies to EBV VCA and EBNA, and to CMV, as well as avidity were tested using Liaision Technologies, according to the manufacturer's instructions (Liaison Technologies, Perugia, Italy).

Viral load for EBV DNA was tested as described by Niesters et al. [18].
Immunohistochemistry: LMP1 expression on BM smears were examined using the Ivew DAB detection kit (Ventana Medical Systems, Inc., Tucson, AZ) using monoclonal mouse anti-Epstein Barr Virus, LMP clones CS 1-4 (DakoCytomation, Denmark), and FISH analysis using the LSI D7S522 (7q31) spectrum orange/CEP spectrum green (VYSIS) probe. More than 800 cells were examined.

Figure 1 depicts the antibody responses to $\operatorname{IgM}, \operatorname{IgG}$ against EBV's viral capsid antigen (VCA), and EBV's nuclear antigen (EBNA) at time intervals from 2 weeks to 6 months after onset of the disease. It shows a three-fold increase in the level (antibody titer) of VCA's IgG antibodies at 6 weeks after disease onset, while the humeral immune response to EBNA, a classic pattern of EBV acute infection, increases about 6 months after disease onset. The IgM response to VCA gradually declined over three months.

Although no EBV DNA could be detected in the first blood sample, which was taken 3 weeks post-disease onset, EBV DNA was present when tested in a nasopharyngeal smear 12 weeks after disease onset as well as in blood samples. EBV viral load at 15, 29, and 42 weeks post-illness onset were $>25,100$, and 100 copies $/ \mathrm{ml}$, respectively.

\subsection{Hematologic Tests}

At admission complete blood count showed mild leukopenia (4400/ul) and neutropenia (900/ul), severe anemia (5.0 g/dl), normal RBC indices, elevated RDW, and normal PLT count. CBC tests performed during the one-year follow up demonstrated mostly leukocyte counts of 4300 - 5600/ $\mu$ l (normal low level of 5000/ul) and neutropenia of $770-1440 / \mu$ l (normal low level of $1500 / \mu \mathrm{l})$. During the first 6 months of treatment the patient received a total of 7 units of packed red blood cells. Following the transfusion of every unit, the $\mathrm{Hb}$ level was raised to 10.0 - $11.0 \mathrm{~g} / \mathrm{dl}$; however, rapidly falling afterwards (Figure 2). After this period the $\mathrm{Hb}$ level gradually and constantly increased without need for additional blood transfusions. PLT count remained normal during ing the one-year follow up period. The first two samples demonstrated mild monocytosis with few immature cells, possibly a result of a reactive process. However, a constant finding during the whole year was mild dysplastic changes, mostly in the erythroid lineage, which included the presence of macronormoblasts, and few bi-nucleated normoblasts or cells with nuclear budding/karyorrhexis. In the megakaryocytic lineage, hypolobularity and/or separate nuclear lobes were detected. Peripheral blood smears examined in parallel with BM aspirates demonstrated mild to moderate anisocytosis with or without the presence of single normoblasts. 


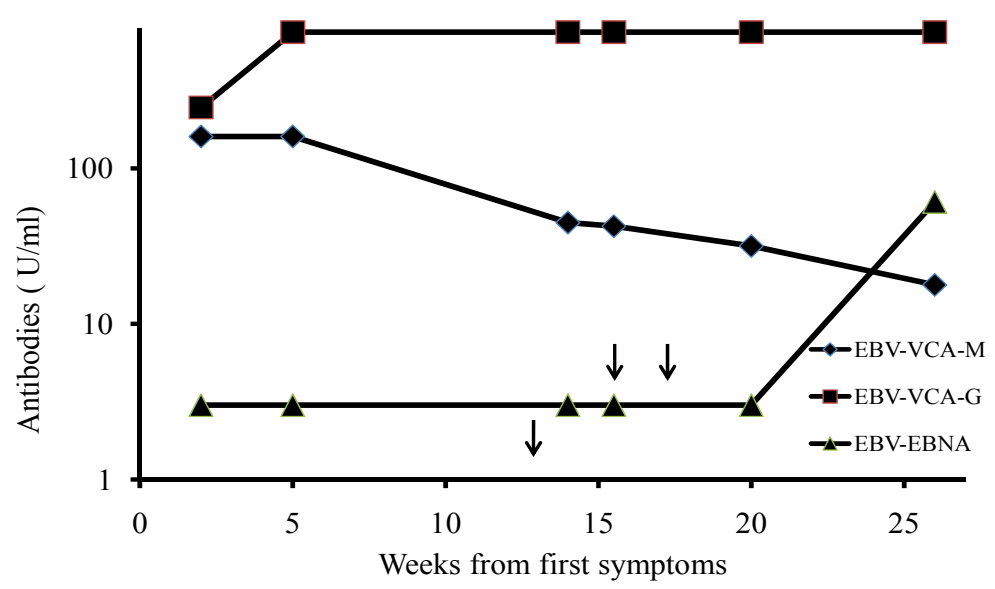

Figure 1. Antibody responses to EBV during the time course of the infection. IgM (diamonds) and IgG (squares) antibodies to VCA, as well as antibodies to EBNA (triangle, EBNA-G) were tested in the patient's sera starting two weeks after initial symptoms. The cut-off values were: VCA-M $>40$, positive; VCA-G/EBNA-G $>20$, positive. Black arrows represent EBV-DNA positive samples, taken from the larynx.

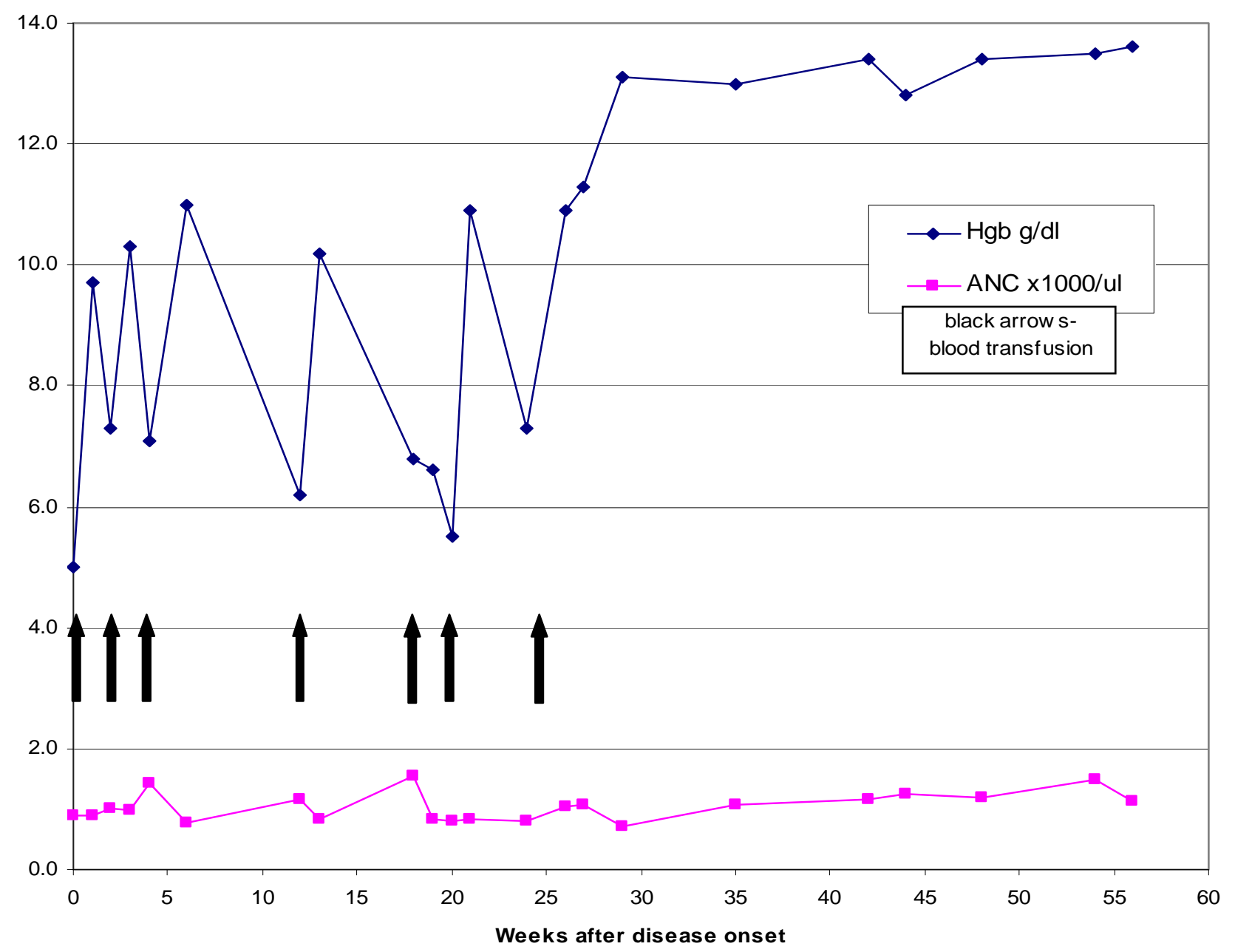

Figure 2. Blood count anemia parameters. Hemoglobin (HB), Hematocrit (HCT), and red blood cell count (RBC) during 40 weeks post-disease onset. Transfusion of packed cells has been given to the patient noted by the arrows. Normal values: Hemoglobin (HB): 11.5 - $13.5 \mathrm{~g} / \mathrm{dl}$, Hematocrit (HCT): 34\% - 40\%, Red blood cells (RBC): $4.2-5.4 \times 10^{6}$. Arrows: transfusion of packed cells. 
Figure 2 shows the results of the absolute neutrophilic count (ANC) and Hemoglobin ( $\mathrm{Hb}$ ) during the 55 weeks post-disease onset. Transfusions of packed cells given to the patient are noted by arrows. No significant abnormalities were found in all other hematology parameters.

\subsection{Cytogenetic Results}

Table 1 summarizes the cytogenetic and FISH results for monosomy 7 in the bone marrow and blood samples. Cytogenetic analysis of the first bone marrow sample revealed monosomy 7 as a sole aberration in all the analyzed dividing cells; FISH analysis revealed that only $57 \%$ of the cells carry monosomy 7 . FISH analysis of the peripheral blood sample showed cells carry monosomy 7 with reduction in the percentage of these cells in the blood in most samples.

Table 1. Monosomy (mo) 7 in blood and bone marrow (BM) samples by cytogenetics and FISH analysis. In parentheses is the number of monosomy 7 cells out of the number of the total analysed cells.

\begin{tabular}{|c|c|c|c|}
\hline Weeks p.d.o. & Sample & Cytogenetics- $\%$ mo-7 & FISH- $\%$ mo-7 \\
\hline 4 & $\mathrm{BM}$ & $100 \%(15 / 15)$ & $57 \%$ \\
\hline 7 & $\mathrm{BM}$ & $70 \%(9 / 13)$ & $36 \%$ \\
\hline 11 & $\mathrm{BM}$ & $50 \%(8 / 16)$ & $26 \%$ \\
\hline 21 & $\mathrm{BM}$ & $70 \%(7 / 10)$ & $18 \%$ \\
\hline 29 & $\mathrm{BM}$ & $80 \%(12 / 15)$ & $30 \%$ \\
\hline 35 & $\mathrm{BM}$ & $26 \%(4 / 11)$ & $19 \%$ \\
\hline 41 & $\mathrm{BM}$ & $75 \%(11 / 15)$ & $30 \%$ \\
\hline 43 & $\mathrm{BM}$ & $75 \%(9 / 12)$ & $34 \%$ \\
\hline 55 & $\mathrm{BM}$ & $75 \%(11 / 15)$ & $34 \%$ \\
\hline Control & $\mathrm{BM}$ & $0 \%$ & $3 \%$ \\
\hline 6 & blood & nd & $34 \%$ \\
\hline 9 & blood & nd & $24 \%$ \\
\hline 13 & blood & nd & $34 \%$ \\
\hline 15 & blood & nd & $22 \%$ \\
\hline 17 & blood & nd & $20 \%$ \\
\hline 19 & blood & nd & $32 \%$ \\
\hline 22 & blood & nd & $15 \%$ \\
\hline 26 & blood & nd & $20 \%$ \\
\hline 29 & blood & nd & $30 \%$ \\
\hline 31 & blood & nd & $17 \%$ \\
\hline 35 & blood & nd & $12 \%$ \\
\hline 41 & blood & nd & $25 \%$ \\
\hline 48 & blood & nd & $20 \%$ \\
\hline 57 & blood & nd & $18 \%$ \\
\hline 60 & blood & nd & $20 \%$ \\
\hline
\end{tabular}

p.d.o.: post disease onset.
There was a decline in the percentage of cells carrying monosomy 7 during the months following disease onset (intervals of 2 - 4 weeks). The percentage of cells carrying monosomy 7 reached a steady level of $20 \%$ to $30 \%$ $( \pm 5 \%)$ in blood samples 3 to 13 months after disease onset. In general, FISH analyses showed decreased levels of monosomy 7 percentage compared to the cytogenetic results of the same BM samples.

\subsection{Immunohistochemical Analysis}

Immunohistochemical staining for LMP1 revealed about 9\% LMP1 positive cells in BM smears, at 4, 7, 11, and 21 weeks post disease onset. Control normal BM smears showed 3\% LMP1 positive cells. Since LMP1 antibodies showed non-EBV-specific staining in young myeloid cells, it was impossible to indicate whether LMP1 was expressed in the stained cells. FISH analysis of the LMP1 stained smears for monosomy 7 showed an increasing percentage of cells carrying monosomy 7 (from $40 \%$ to $80 \%$ ) in the stained cells (Figure 3). The LMP1 positive cells showed a neutrophil morphology.

\section{DISCUSSION}

We described here a 4-year-old girl suffering from simultaneous acute EBV infection and refractory anemia MDS with chromosome 7 monosomy. The main question arising is whether the MDS is caused by the EBV infection, as either a direct modulator or an indirect modulator (cofactor), or whether this is a case of coincidence in which the two diseases occurred independently at the same time.

Infectious agents, particularly viruses, are known to play an important role in human cancer development. Recent estimates have shown that $17.8 \%$ of cancer cases are attributable to infectious agents, and of these $12.1 \%$ are caused by viral infections [19].

Epstein Barr Virus (EBV) is one of the causative agents of infectious mononucleosis and the first described human tumor virus found to be associated with Burkitt's lymphoma tumor characterized by $\mathrm{t}(8,14), \mathrm{t}(2,8)$ and $\mathrm{t}(8,2)$ [10], Hodgkin's lymphoma, nasopharyngeal carcinoma, post-transplant lymphoproliferative disorders (PTLD), and more $[11,12,20]$.

Apparently, viral gene products play an active role in the pathogenesis of these diseases, [12] disrupting the balance of antiviral immunity leading to the development of EBV-associated disease [11].

The latent membrane protein (LMP1) gene of EBV is considered the main viral oncogene driving cell growth, promoting metastases, apoptotic resistance, and immune modulation including blocking interferon alpha-induced antiviral signaling [13]. In most EBV-associated tumors including PTLD, HD, and NPC, LMP1 protein is rela- 

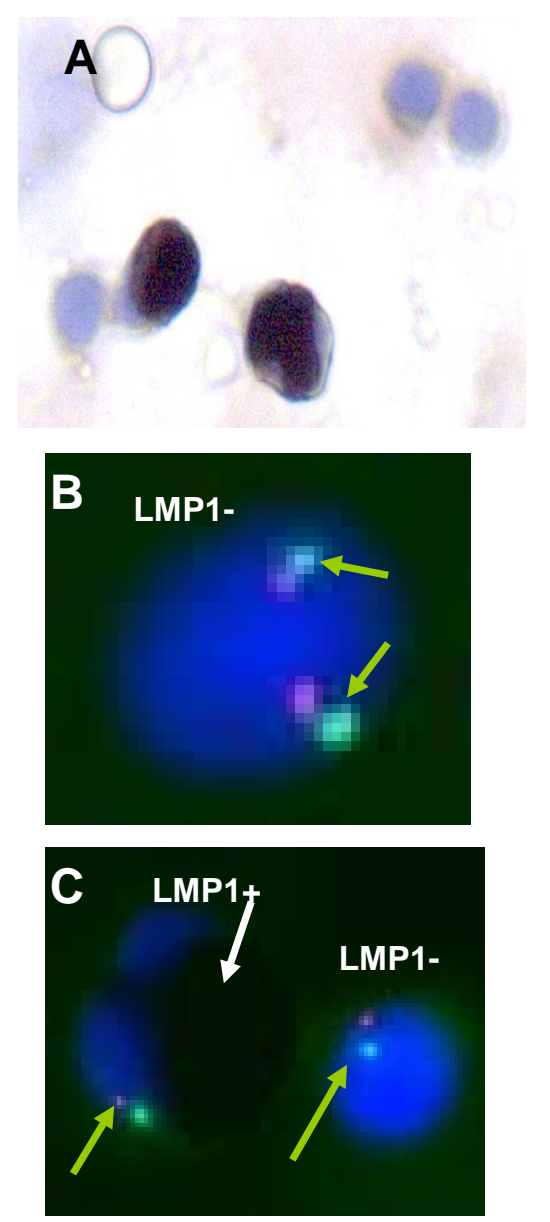

Figure 3. Immunohistochemistry using LMP1 antibodies and FISH analysis for chromosome 7 of the BM smears. (A) LMP1 positive and negative cells in a BM smear (400×); (B) LMP1 negative cell with two chromosome 7 signals; (C) Positive and negative cells with one signal of chromosome 7 (LSI D7S522 (7q31) spectrum orange/CEP spectrum green (VYSIS) probe) $(1000 \times)$.

tively highly expressed [13,21-23]. Moreover, in immuno compromised patients abundant LMP1 expression can be observed in tonsillar B cells prior to onset of lymphoproliferative disease [24].

Myelodysplastic syndromes (MDS) are characterized by ineffective hematopoiesis and a high propensity to transform to acute myeloid leukemia (AML). These are common hematological diseases in the elderly but are rarely observed in children [25]. The etiology and molecular pathogenesis of MDS are still poorly understood [26]. Both gene mutations and cytogenetic changes play important roles in the pathogenesis of this disease. The main genetic diagnosis of MDS so far is by karyotyping [27]; monosomy 7 is one of the common chromosomal abnormalities in MDS. The outcome of monosomy 7
MDS in children is often rather poor [28] and the treatment in such cases is BM transplantation.

Cytogenetic, histologic, and virologic analyses of the present patient were consistent with MDS, a subtype refractory anemia associated with $\mathrm{EBV}$ infection.

Two patterns of monosomy 7 MDS in children are described in the literature. One is the sporadic monosomy 7 MDS characterized by a striking sex difference, with males affected 3 - 10 times more than females $[4,29]$; median age at disease onset is relatively young ( 3 years). The second is the familial monosomy 7 MDS with no sex preference and with later onset of the disease at a median age of $10-14$ years $[9,28,30]$. The inheritance mode of both MDS patterns is thought to be polygenic-mulifactorial.

The described patient is most probably a sporadic MDS case, as there was no evidence for familial monosomy 7 MDS. The patient's age is close to the median age for sporadic MDS. However, the patient is a female in contrast to the male prevalence in sporadic MDS. In polygenic multifactorial inheritance this strengthens the possibility of prominent genetic involvement. The patient's high susceptibility to bacterial and viral infections may also strengthen the possibility of a genetic predisposion for leukemia disorder development.

The virology results showed a high titer of VCA IgM and $\mathrm{IgG}$ at 2 weeks after EBV infection symptoms onset (on hospital admittance) followed by an increase of EBNA IgG titer together with EBV DNA positive nasopharingeal smear, as well as in blood samples, further strengthening the diagnosis of EBV acute infection. The presence of $<25-100$ copies/ml of EBV in blood samples during the 55 weeks post-infection, may reflect the latent stage of the EBV in the patient.

Although CMV IgM and IgG were positive in the patient's blood, the high avidity points strongly towards reactivation response. Also, CMV is known to be less tumorogenic compared to EBV.

The patient received supportive care of transfusions of packed cells during the 24 weeks post-disease onset with no antileukemic therapy (Figure 2). The patient is a candidate for BM transplantation.

Cytogenetic and FISH analyses follow-up of monosomy 7 percentage in bone marrow and peipheral blood samples showed, in general, a decrease in the percentage of cells carrying monosomy 7 during the 13 months after disease onset. This is an original observation.

Table 1 shows a discrepancy between the cytogenetics and FISH analysis regarding monosomy 7 percentage. While in cytogenetics monosomy 7 was the sole aberration in all the cells, FISH analysis revealed only 57\% monosomy 7 . This most probably indicates a higher rate of cell division (examined by cytogenetics) in the ab- 
normal cells compared with the normal cells. We found a similar discrepancy when other FISH probes were used (7q13, 5q33, and centromere 8-personal experience, unpublished data). These results are in agreement with those of Harrison et al., [31] showing that in some cases there is preference for the cell carrying the chromosomal abnormality to divide, compared to normal cells.

Immunohistochemistry of the BM smears using antibodies against EBV LMP1 antigen showed 9\% LMP1 positive cells in all examined samples. FISH analysis of the LMP1 stained smears for monosomy 7 showed an increase in the percentage of the LMP1 positive cells carrying monosomy 7 . We could not differentiate between the specific and non-specific EBV LMP1 staining since the LMP1 antibodies used in this study cross-react with young myeloid cells [32]. Nevertheless, according to their morphology, LMP1 positive cells were neutronphils. The significance of the increasing LMP1 positive cell percentage carrying monosomy 7 is unclear.

As far as we know there are no reports on pediatric MDS caused by EBV infection. However, there are some reports that point to the possibility that EBV plays an important role in MDS or leukemia development. Mahoney et al. [33] described persistent EBV infection coinciding with the evolution of myelodysplasia in a 5-year-old child. Manabe et al. [34] showed an association between reactivation of EBV infection and juvenile myelomonocytic leukemia (JMML) in 4 patients; Stollman et al. [35] described a child with persistent EBV infection and a $\mathrm{t}(3,5)$-positive myeloproliferative disease resembling JMML.

Moreover, in patients with chronic active EBV infection, which can evolve to virus-associated hemophagocytic syndrome [36], the peripheral blood $\mathrm{T}$ cells and natural killer cells showed monoclonal integration of the EBV genome [37], a hallmark of natural killer cell-associated leukemia/lymphoma. Recently, Borze et al. [38] showed that EBV miRNA ebv-miR-BART13 was upregulated in 19 cases of MDS. All the above reports support the possibility that EBV plays an important role in MDS development.

\section{CONCLUSION}

The simultanous occurrence of both diseases is convincing that they are related. Our results suggest that EBV can be either the cause of the MDS development or a crucial co-factor in its development. It is also possible that a pre-existing subclinical MDS became apparent at time of a primary EBV-infection due to additional myelosuppression or was promoted by virus-induced immunomodulation. The possibility that EBV or other viruses contribute to MDS pathogenesis by stimulating a preexisting clone or pre-existing genetic predisposition to develop myeloproliferative disorders warrants further investigation.

\section{REFERENCES}

[1] Niemeyer, C.M. and Baumann, I. (2008) Myelodysplastic syndrome in children and adolescence. Seminars in Hematology, 45, 60-70. doi:10.1053/j.seminhematol.2007.10.006

[2] Mitelman, F. and Heim, S. (1992) Quantitative acute leukemia cytogenetics. Genes Chromosomes \& Cancer, 5, $57-$ 66. doi: $10.1002 / \mathrm{gcc} .2870050109$

[3] Johansson, B., Mertens, F. and Mitelman, F. (1993) Cytogenetic deletion maps of hematologic neoplasms; Circumstantial evidence for tumor suppressor loci. Genes Chromosomes \& Cancer, 8, 205-218. doi:10.1002/gcc. 2870080402

[4] Luna-Fineman, S., Shannon, K.M. and Lange, B.J. (1995) Childhood monosomy 7: Epidemyology, biology and mechanistic implications. Blood, 85, 1985-1999.

[5] Sieff, C., Chessells, J., Harvey, A., et al. (1981) Monosomy 7 in childhood: A myeloproliferative disorder. British Journal of Haematology, 49, 235-249. doi:10.1111/j.1365-2141.1981.tb07220.x

[6] Hasle, H., Arico, M., Basso, G., et al. (1999) Myelodysplastic syndrom, juvenile myelomonocytic leukemia, and acute myeloid leukemia associated with complete or partial monosomy 7. Leukemia, 13, 376-385. doi:10.1038/sj.leu.2401342

[7] Kardos, G., Bumann, I., Passmore, S.I., et al. (2003) Refractory anemia in childhood: A retrospective analysis of 67 patients with particular reference to monosomy 7 . Blood, 102, 1997-2003. doi:10.1182/blood-2002-11-3444

[8] Johnson, E. and Cotter, F. (1997) Monosomy 7 and 7qassociated with myeloid malignancy. Blood Reviews, 11, 46-55. doi:10.1016/S0268-960X(97)90006-0

[9] Hall, G.W. (2001) Childhood myeloid leukemias. Best Practice \& Research Clinical Haematology, 14, 573-591. doi:10.1053/beha.2001.0155

[10] Epstein, M.A., Achong, B.G. and Barr, S.Y.M. (1964) Virus particles in cultured lymphoblasts from Burkitt's lymphoma. Lancet, 1, 702-703. doi:10.1016/S0140-6736(64)91524-7

[11] Williams, H. and Crawford, D.H. (2006) Epstein-Barr virus: The impact of scientific advances on clinical practice. Blood, 107, 862-869. doi:10.1182/blood-2005-07-2702

[12] Middeldorp, J.M., Brink, A.A., van den Brule, A.J., et al. (2003) Pathogenic roles for Epstein-Bar virus (EBV) gene products in EBV-associated proliferative disorders. Critical Reviews in Oncology Hematology, 45, 1-36. doi:10.1016/S1040-8428(02)00078-1

[13] Geiger, T.R. and Martin, J.M. (2006) The Epstein-Barr virus-encoded LMP-1 oncoprotein negatively affects Tyk2 phosphorylation and interferon signaling in human B cells. Jounal of Virology, 80, 11638-11650. doi:10.1128/JVI.01570-06

[14] Herrod, H., Dow, L. and Sullivan, J. (1983) Persitant 
Epstien Barr virus infection mimicking juvenile chronic myelogenous leukemia: Immunologic and hemathologic studies. Blood, 61, 1098-1104.

[15] Kirby, M.A., Weitzman, S. and Freedman, N.H. (1990) Juvenile chronic myelogenous leukemia: Differentiation from infantile cytomegalovirus infection. Journal of Pediatric Hematology Oncology, 12, 292-296. doi:10.1097/00043426-199023000-00007

[16] Lorenzan, L., Lyons, H., Sawaf, H., et al. (2002) Human herpes virus 6 infection mimicking juvenile chronic myelogenous leukemia in an infant. Journal of Pediatric Hematology Oncology, 24, 136-141. doi:10.1097/00043426-200202000-00016

[17] Shaffer, L.G., Slovak, M.L. and Campbell, L.J., Eds. (2009) An international system for human cytogenetic nomenclature. S. Karger, Basel.

[18] Niesters, H.G., Van Esser, J., Fries, E., et al. (2000) Development of a real-time quantitative assay for detection of Epstein-Barr virus. Journal of Clinical Microbiology, 38, 712-715.

[19] Parkin, D.M. (2003) The global health burden of infection-associated cancers in the year 2002 (review). International Journal of Cancer, 118, 3030-3040. doi:10.1002/ijc. 21731

[20] Cohen, J.I. (2000) Epstein-Barr virus infection. New England Journal of Medicine, 343, 481-492. doi:10.1056/NEJM200008173430707

[21] Leibowitz, D. (1998) Epstein-Bar virus and a cellular signaling pathway in lymphomas from immunosuppressed patients. New England Journal of Medicine, 338, 14131421. doi:10.1056/NEJM199805143382003

[22] Dukers, D.F., Jaspars, L.H., Vos, W., et al. (2000) Quantitative immunohistochemical analysis of cytokine profiles in Epstein-Barr virus-positive and negative cases of Hodgkin's disease. Journal of Pathology, 190, 143-149. doi:10.1002/(SICI)1096-9896(200002)190:2<143::AID-P ATH519>3.0.CO;2-5

[23] Khabir, A., Karray, H., Rodriguez, S., et al. (2005) EBV latent membrane protein 1 abundance correlates with patient age but not with metastatic behavior in north African nasopharyngeal carcinomas. Virology Journal, 2, 39. doi:10.1186/1743-422X-2-39

[24] Mowry, S.E., Strocker, A.M., Chan, J., et al. (2008) Immunohistochemical analysis and Epstein-Barr virus in the tonsils of transplant recipients and healthy controls. Archives of Otolaryngology-Head \& Neck Surgery, 134, 936-939. doi:10.1001/archotol.134.9.936

[25] Hasle, H. and Niemeyer, C.M. (2002) Myelodysplastic syndrome and juvenile myelomonocytic leukemia in children. In: Bennett, J.M., Ed., The Myelodysplastic Syndromes: Pathology and Clinical Management, Marcel Dekker Inc., New York, 299-344.

[26] Platzbecker, M., Meredyth-Stewart, M. and Eninger, G. (2007) The pathogenesis of meylodysplatic syndromes (MDS). Cancer Treatment Reviews, 33, S53-S58. doi:10.1016/j.ctrv.2007.07.021

[27] Bejar, R. and Ebert, B.L. (2010) The genetic basis of myelodysplatic syndromes. Hematology-Oncology Clinics of North America, 24, 295-315. doi:10.1016/j.hoc.2010.02.001

[28] Kwong, Y.L., Ng, M.H. and Ma, S.K. (2000) Familial acute myeloid leukemia with monosomy 7: Late onset and involvement of a multipotential progenitor cell. Cancer Genetics and Cytogenetics, 116, 170-173. doi:10.1016/S0165-4608(99)00121-1

[29] Kwong, Y.L. and Chan, L.C. (1994) Involvement of eosinophils in acute myeloid leukemia with monosomy 7 demonstrated by in situ hybridization. British Journal of Haematology, 88, 389-391. doi:10.1111/j.1365-2141.1994.tb05035.X

[30] Shannon, K.M., Turhan, A.G., Rogers, P.C.J., et al. (1992) Evidence implicating heterozygous deletion of chromosome 7 in the pathogenesis of familial leukemia associated with monosomy 7. Genomics, 80, 332-336.

[31] Harrison, K.J., Massing, B., McKenna, C., et al. (1995) Molecular cytogenetic analysis of monosomy 7 in pediatric patients with myelodysplastic syndrome. American Journal of Hematology, 48, 88-91. doi:10.1002/ajh.2830480204

[32] Leong, A.S.Y., Cooper, K., Joel, F. and Leong, W.M. (1999) Manual of diagnostic antibodies for immunohistology. Oxford University Press, Oxford, 162.

[33] Mahoney Jr., D.H., McClain, K.L., Hanson, I.C., et al. (1989) Acquired immune deficiency, myelodysplasia and acute nonlymphocytic leukemia associated with monosomy 7 and $\mathrm{t}(3 ; 3)$ (q21;q26) in a child with langerhans cell histocytosis. American Journal of Pediatric Hematology, 11, 153-157.

[34] Manabe, A., Yoshimasu, T., Ebihara, Y., et al. (2004) Viral infection in juvenile myelomonocytic leukemia: Prevalence and clinical implications. Journal of Pediatric Hematology Oncology, 26, 636-641. doi:10.1097/01.mph.0000140653.50344.5c

[35] Stollmann, B., Fonatsch, C.H. and Havers, W. (1985) Persistent Epstein-Barr virus infection associated with monosomy 7 or chromosome 3 abnormality in childhood myeloproliferative disorders. British Journal of Haematology, 60, 183-196. doi:10.1111/j.1365-2141.1985.tb07399.x

[36] Straus, S.E. (1998) The chronic mononucleosis syndrome. Journal of Infectious Diseases, 157, 280-286.

[37] Kimura, H., Hohino, Y., Kanegane, H., et al. (2001) Clinical and virologic characteristics of chronic Epstein-Barr virus infection. Blood, 98, 280-286. doi:10.1182/blood.V98.2.280

[38] Borze, I., Scheinin, I., Sitonen, S., et al. (2011) miRNA expression profiles in myelodysplastic syndromes reveal Epstein-Barr virus miR-BART 13 dysregulation. Leukemia \& Lymphoma, 52, 1567-1573. doi: $10.3109 / 10428194.2011 .568652$ 